\title{
Adjusting the STEM Sample Holder Potential for Improved EBIC Contrast
}

\author{
Matthew Mecklenburg ${ }^{1 *}$, William A. Hubbard ${ }^{2,3}$, Ho Leung Chan ${ }^{2,3}$, and B. C. Regan ${ }^{2,3}$ \\ ${ }^{1 .}$ Core Center of Excellence in Nano Imaging, University of Southern California, Los Angeles, USA. \\ 2. Department of Physics and Astronomy, University of California, Los Angeles, USA. \\ 3. California NanoSystems Institute, University of California, Los Angeles, USA. \\ * Corresponding author: matthew.mecklenburg@usc.edu
}

Here we show that by adjusting the voltage between the pole piece and the sample holder in a scanning transmission electron microscope (STEM) we can modify the contrast generated by secondary electron (SE) emission. Imaging using SEs provides a signal related to a sample's topology and electronic work function. Recently, we have developed a method of detecting SEs as an electron beam-induced current (EBIC) [1]. This detection method is also sensitive to traditional EBIC signals such as electron-hole pair generation in local electric fields [2].

Of primary electrons with energies typical for TEM $(80-300 \mathrm{keV})$, only a few percent yield secondaries [1]. Usually an SE detector, such as an ETD [3-5], is used to detect a portion the SEs emitted from the top (i.e. beam-incident) side of the sample. In TEM both the top and bottom sides emit SEs as the primary beam travels into and out of the sample. Using SEEBIC we detect the total emission of electrons from both sides (Fig. 1). A primary current of a few hundred pA generates an EBIC of a few $\mathrm{pA}[1]$.

Adjusting potentials near the sample can significantly change the SE signal contrast [1,4,5]; SEs have only a few $\mathrm{eV}$ of kinetic energy and are therefore strongly influenced by local electric and magnetic fields. For example, SEs emitted into an electric field directed away from the emission point can be recaptured, thereby diminishing the detected SEEBIC. TEM manufacturers apply a bias voltage between the sample holder and the pole piece (which is electrically connected to the TEM column) to trigger a pole-touch alarm: when the holder and pole piece touch, a current flows and sets off the alarm. By default, the bias on the holder, relative to the pole piece, is typically $-5 \mathrm{~V}$ in JEOL microscopes and $2 \mathrm{~V}$ in ThermoFischer/FEI microscopes - these potentials are more than sufficient to alter the SE recapture rate [1]. Here we have disabled the alarm and inserted an adjustable voltage source.

Adjusting the potential between the holder and the pole piece dramatically alters the SEEBIC (Fig. 2). Making the holder potential more positive, relative to the pole piece, attracts low energy SEs back to the holder, decreasing the SEEBIC signal. The small contrast change between the $+10 \mathrm{~V}$ and $+20 \mathrm{~V}$ images indicates that most of the SEs have energy less than $10 \mathrm{eV}$. A negative holder potential leads to an increase in signal, as the local electric field encourages SEs to escape. The bright and dark SEEBIC features in the thicker regions of the sample that persist even for large holder bias are likely due to processes (e.g. beam absorption, emission of SE from backscattered electrons) that occur less frequently in electron-transparent regions of the sample [1]. No bias-induced changes are visible in the Fig. 2 ADF STEM images.

The ability to control the potential between the holder and the pole piece not only provides a spectroscopic handle on the emitted SE, but may also facilitate the measurement of work functions [1]. Moreover, it might also allow the mitigation of charging in insulating samples [6]. 


\section{References:}

[1] WA Hubbard et al., Physical Review Applied 10 (2018), 044066.

[2] E White et al., Applied Physics Letters 107 (2015), 223104.

[3] TE Everhart and RFM Thornley, Journal of Scientific Instruments 37 (1960), p. 246.

[4] H Inada et al., Ultramicroscopy 111 (2011), p. 865.

[5] CA Walsh, Ultramicroscopy 45 (1992), p 85.

[6] The authors would like to thank Claude Kroupa, Robert Bent, and Nathan Didier from JEOL for their help with accessing and modifying the sample holder biasing circuit. Data presented here were acquired at the Core Center of Excellence in Nano Imaging (CNI) at the University of Southern California. This work was supported by National Science Foundation (NSF) award DMR-1611036 and by NSF STC award DMR-1548924 (STROBE).
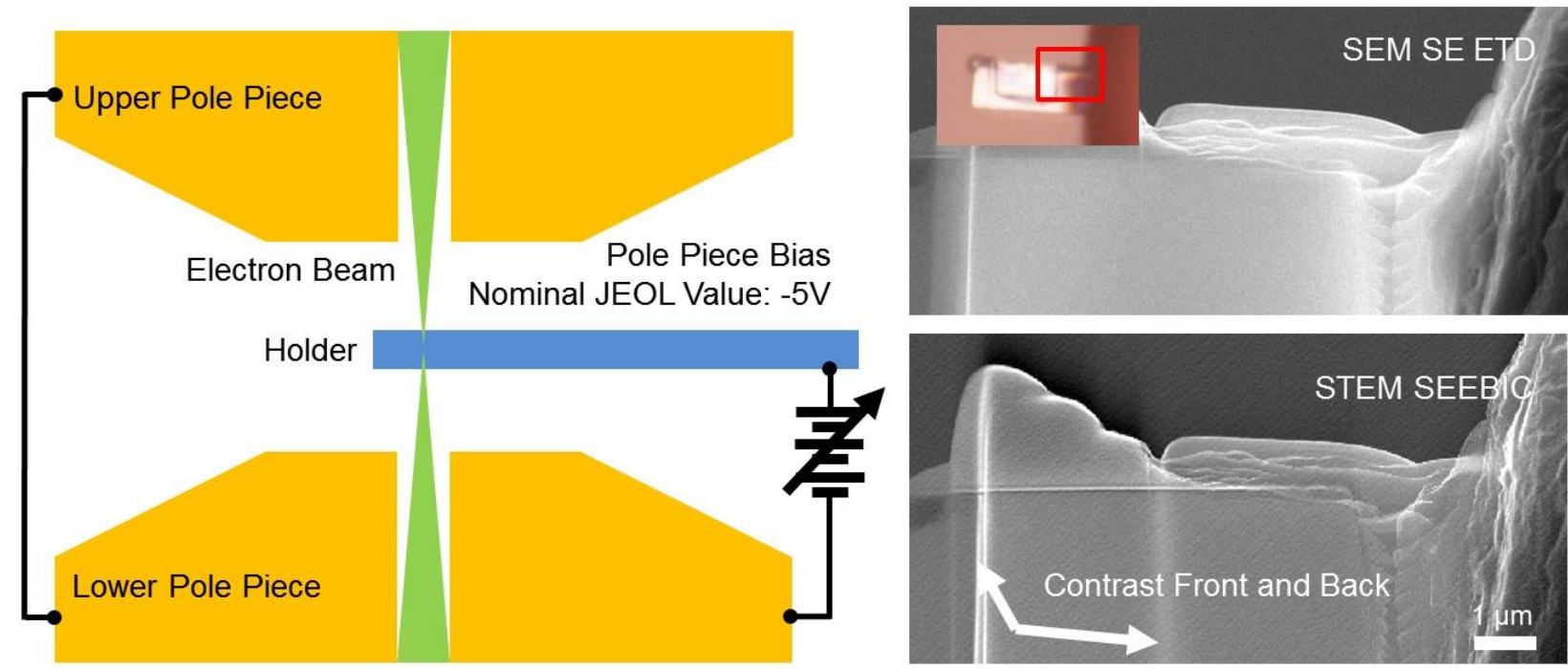

Figure 1. (left) Pole piece and bias voltage geometry. FIB-prepared lamella (optical image inset, ROI selected) imaged with an Everhart-Thornley detector (ETD) in an SEM (right top), and with SEEBIC in a STEM (right bottom).
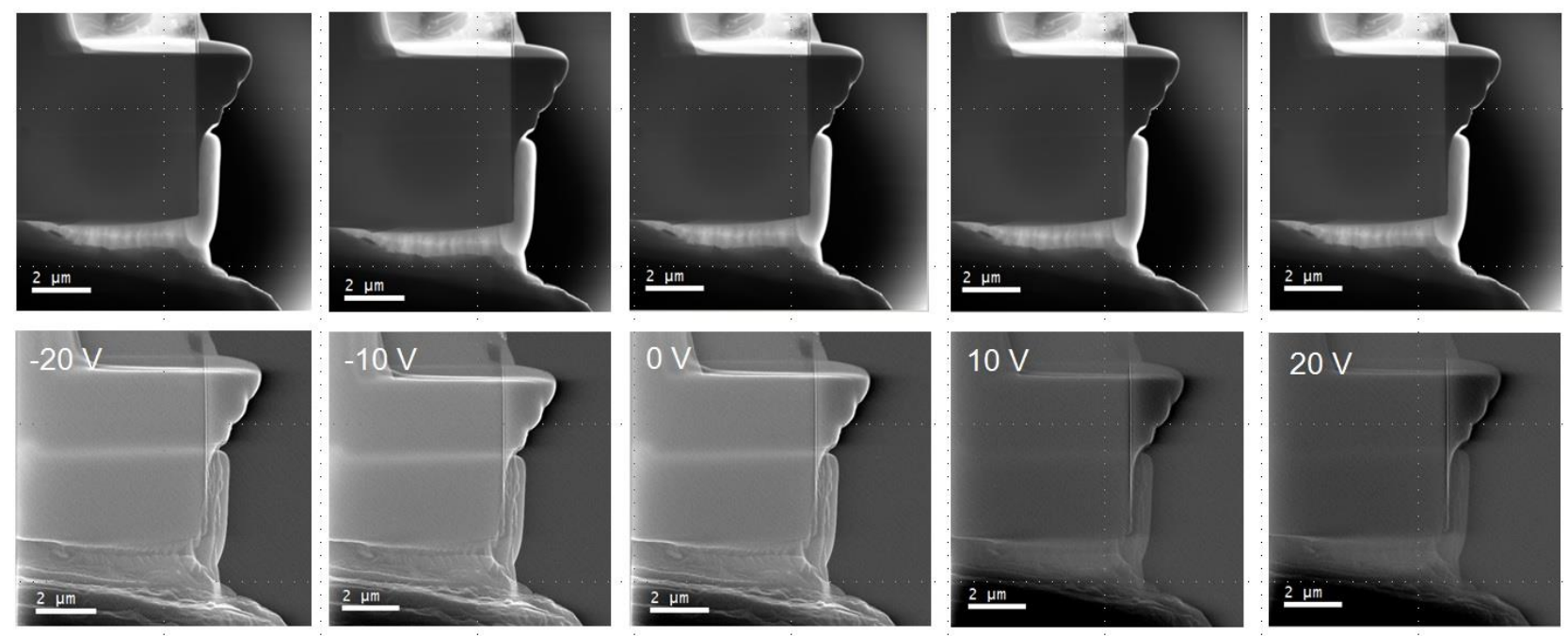

Figure 2. STEM annular dark field (ADF, top row) and SEEBIC (bottom row) images at different holder bias values relative to the pole piece (as indicated in SEEBIC images). 